JOURNAL OF

SYMPLECTIC GEOMETRY

Volume 4, Number 1, 93-116, 2006

\title{
CONTACT STRUCTURES ON OPEN 3-MANIFOLDS
}

\author{
JAMES J. TRIPP
}

In this paper, we study contact structures on any open 3-manifold $V$ that is the interior of a compact 3-manifold. To do this, we introduce new proper contact isotopy invariants called the slope at infinity and the division number at infinity. We first prove several classification theorems for $T^{2} \times[0, \infty), T^{2} \times \mathbb{R}$, and $S^{1} \times \mathbb{R}^{2}$ using these concepts. The only other classification result on an open 3-manifold is Eliashberg's classification on $\mathbb{R}^{3}$. Our investigation uncovers a new phenomenon in contact geometry: There are infinitely many tight contact structures on $T^{2} \times[0,1)$ that cannot be extended to a tight contact structure on $T^{2} \times[0, \infty)$. Similar results hold for $T^{2} \times \mathbb{R}$ and $S^{1} \times \mathbb{R}^{2}$. Finally, we show that if every $S^{2} \subset V$ bounds a ball or an $S^{2}$ end, then there are uncountably many tight contact structures on $V$ that are not contactomorphic, yet are isotopic. Similarly, there are uncountably many overtwisted contact structures on $V$ that are not contactomorphic, yet are isotopic. These uncountability results generalize work by Eliashberg when $V=S^{1} \times \mathbb{R}^{2}$.

\section{Introduction}

Recently, there has been much work towards the classification of tight contact structures on compact 3-manifolds up to isotopy (relative to the boundary). In particular, Honda and Giroux provided several classification theorems for solid tori, toric annuli, torus bundles over the circle, and circle bundles over surfaces $[\mathbf{8}, \mathbf{9}, \mathbf{1 0}, \mathbf{1 3}, \mathbf{1 4}]$. In comparison, tight contact structures on open 3-manifolds have been virtually unstudied. Two main results dealing with open contact manifolds are due to Eliashberg. In [3], Eliashberg shows that $\mathbb{R}^{3}$ has a unique tight contact structure. It is immediate from his proof that $S^{2} \times[0, \infty)$ has a unique tight contact structure with a fixed characteristic foliation on $S^{2} \times 0$. Therefore, the classification of tight contact structures on open manifolds with only $S^{2}$ ends can be reduced to the case 
of compact manifolds. In [5], Eliashberg shows that, in contrast to the situation for $S^{2}$ ends, there are uncountably many tight contact structures on $S^{1} \times \mathbb{R}^{2}$ that are not contactomorphic. The situation for closed 3-manifolds is different. Colin et al. [2] proved that an atoroidal 3-manifold supports finitely many tight contact structures up to isotopy. Honda et al. [15], and independently, Colin [1], show that an irreducible, toroidal 3-manifold supports countably infinitely many tight contact structures up to isotopy.

In this paper, we study tight contact structures on any open 3-manifold $V$ that is the interior of a compact manifold. Due to the failure of Gray's theorem on open contact manifolds, we relegate ourselves to the study of tight contact structures up to proper isotopy, by which we mean isotopy of the underlying manifold rather than a one-parameter family of contact structures. When we say that two contact structures are isotopic, we will mean that they are connected by a one-parameter family of contact structures. We first introduce two new proper isotopy invariants which we call the slope at infinity and the division number at infinity of an end $\Sigma_{g} \times[0, \infty)$ of an open contact manifold. These invariants are most naturally defined for toric ends $T^{2} \times[0, \infty)$, where we take our inspiration from the usual definition of the slope and division number of a convex torus. Using these invariants and Honda's work in [13], we essentially classify tight contact structures on toric ends $T^{2} \times[0, \infty)$. In particular, we show that there is a natural bijection between tight toric annuli and tight toric ends that attain the slope at infinity and have finite division number at infinity. However, we also show that for any slope at infinity, there is an infinite family of tight toric ends which do not attain the slope at infinity and therefore do not come from closed toric annuli. This yields the following:

Theorem 1.1. Let $X$ be $T^{2} \times[0,1), T^{2} \times(0,1)$, or $S^{1} \times D^{2}$, where $D^{2}$ is the open unit disk. Let $X^{\prime}$ be another copy of $X$ parametrized as $T^{2} \times[0, \infty)$, $T^{2} \times \mathbb{R}$, or $S^{1} \times \mathbb{R}^{2}$. For each slope at infinity, there exist infinitely many tight contact structures on $X$ with that slope, distinct up to proper isotopy, which do not extend to a tight contact structure on $X^{\prime}$.

This result stands in contrast to Eliashberg's original examples. Eliashberg's examples are all $S^{1} \times D^{2}$ neighborhoods of a transverse curve in the standard tight contact structure on $S^{3}$, and each has a different slope at infinity. Moreover, for each example, one can find an $S^{1} \times \mathbb{R}^{2}$ neighborhood inside $S^{3}$ that extends the $S^{1} \times D^{2}$ neighborhood of the transverse curve. Theorem 1.1 shows that, in general, such an extension need not exist. Indeed, for each slope at infinity, there are infinitely many distinct contact structures that are not extendible to a tight contact structure.

Finally, just as high torus division number is a problem in the classification of toric annuli, contact structures with infinite division number at infinity prove difficult to understand. However, we are able to use the notion of stable 
disk equivalence to partially understand this situation. Precise statements of all of these results are in Section 4. In Section 5, we use these results to reduce the classification of tight contact structures on $S^{1} \times \mathbb{R}^{2}$ and $T^{2} \times \mathbb{R}$ to the classification of the corresponding toric ends.

In the second half of the paper, we use the slope at infinity to study contact structures on any open 3-manifold $V$ that is the interior of a compact manifold. We generalize Eliashberg's results for $V=S^{1} \times \mathbb{R}^{2}$ proved in [5]:

Theorem 1.2. Let $V$ be any open 3-manifold which is the interior of a compact, connected 3-manifold $M$ with nonempty boundary such that every embedded $S^{2}$ either bounds a ball or is isotopic to a component of $\partial M$. If $\partial M$ contains at least one component of nonzero genus, then $V$ supports uncountably many tight contact structures which are not contactomorphic, yet are isotopic.

Eliashberg's proof involves computing the contact shape of the contact structures on $S^{1} \times \mathbb{R}^{2}$, which in turn relies on a previous computation of the symplectic shape of certain subsets of $T^{n} \times \mathbb{R}^{n}$ done in [18]. We bypass the technical difficulties of computing the symplectic shape by employing convex surface theory in the end of $V$. The first step in the proof is to put a tight contact structure on the manifold $M$ with a certain dividing curve configuration on the boundary. To do this, we use the correspondence between taut sutured manifolds and tight contact structures covered in [16]. We then find nested sequences of surfaces which allow us to construct a contact manifold $\left(V, \eta_{s}\right)$ for every $s \in(-2,-1)$. We distinguish these contact structures up to proper isotopy by showing that they have different slopes at infinity. Since the mapping class group of an irreducible 3-manifold with boundary is countable (see $[\mathbf{1 7}]$ ), uncountably many of the $\eta_{s}$ are not contactomorphic. To simplify the presentation of the proof, we first present the proof in the case when $\partial M$ is connected in Section 7.2. We deal with the case of disconnected boundary in Section 7.3.

In [3], Eliashberg declares a contact structure on an open 3-manifold $V$ to be overtwisted at infinity if for every relatively compact $U \subset V$, each noncompact component of $V \backslash U$ is overtwisted. If the contact structure is tight outside of a compact set, then it is tight at infinity. He then uses his classification for overtwisted contact structures in [4] to show that any two contact structures that are overtwisted at infinity and homotopic as plane fields are properly isotopic. In contrast to this result, we have the following:

Theorem 1.3. Let $V$ be any open 3-manifold which is the interior of a compact, connected 3-manifold $M$ with nonempty boundary such that every embedded $S^{2}$ either bounds a ball or is isotopic to a component of $\partial M$. If $\partial M$ contains at least one component of nonzero genus, then $V$ supports uncountably many overtwisted contact structures which are tight at infinity and which are not contactomorphic, yet are isotopic. 


\section{Background and conventions}

For general facts about 3-manifolds, we refer the reader to [11]. For terminoloy and facts about contact geometry and especially convex surface theory, we refer to $[\mathbf{1 3}]$ and $[\mathbf{6}]$. In particular, for the construction of the Farey graph and the relationship to convex tori, see [13]. Given a convex surface $S$ in a contact 3-manifold, we denote the dividing set of $S$ by $\Gamma_{S}$. The Legendrian realization principle (see [13]) says that any nonisolating collection of arcs and closed curves on a convex surface can be made Legendrian after an isotopy of the surface. When we say "LeRP", we will mean "apply the Legendrian realization principle" to a collection of curves. We will use this as a verb and call this process "LeRPing" a collection of curves.

We first review the definition of a net, which we use in the definition of the slope at infinity and the division number at infinity. Let $A$ be a directed set, and let $X$ be a topological space. A net in $\mathrm{X}$ is a function $f: A \rightarrow X$. Let $Y \subset X$. We say that $f$ is eventually in $Y$ if there exists a $\beta \in A$ such that, for all $\alpha \geq \beta, f(\alpha) \in Y$. We say that the net $f$ converges to $x \in X$ if, for every neighborhood $U$ of $x, f$ is eventually in $U$.

We now list some of the definitions and results in $[\mathbf{1 6}]$ which we will need later. A sutured manifold $(M, \gamma)$ is a compact oriented 3-manifold $M$ together with a set $\gamma \subset \partial M$ of pairwise disjoint annuli $A(\gamma)$ and tori $T(\gamma)$. $R(\gamma)$ denotes $\partial M \backslash \operatorname{int}(\gamma)$. Each component of $R(\gamma)$ is oriented. $R_{+}(\gamma)$ is defined to be those components of $R(\gamma)$ whose normal vectors point out of $M$, and $R_{\gamma}$ is defined to be $R(\gamma) \backslash R_{+}(\gamma)$. Each component of $A(\gamma)$ contains a suture which is a homologically nontrivial, oriented simple, closed curve. The set of sutures is denoted $s(\gamma)$. The orientation on $R_{+}(\gamma), R_{-}(\gamma)$, and $s(\gamma)$ are related as follows. If $\alpha \subset \partial M$ is an oriented arc with $\partial \alpha \subset R(\gamma)$ that intersects $s(\gamma)$ transversely in a single point and if $s(\gamma) \cdot \alpha=1$, then $\alpha$ must start in $R_{+}(\gamma)$ and end in $R_{-}(\gamma)$.

A sutured manifold with annular sutures is a sutured manifold $(M, \gamma)$ such that $\partial M$ is nonempty, every component of $\gamma$ is an annulus, and each component of $\partial M$ contains a suture. A sutured manifold $(M, \gamma)$ with annular sutures determines an associated convex structure $(M, \Gamma)$, where $\Gamma=s(\gamma)$. For more on this correspondence, see [16].

A transversely oriented codimension- 1 foliation $\mathcal{F}$ is carried by $(M, \gamma)$ if $\mathcal{F}$ is transverse to $\gamma$ and tangent to $R(\gamma)$ with the normal direction pointing outward along $R_{+}(\gamma)$ and inward along $R_{-}(\gamma)$, and $\left.\mathcal{F}\right|_{\gamma}$ has no Reeb components. $\mathcal{F}$ is taut if each leaf intersects some closed curve or properly embedded arc connecting $R_{-}(\gamma)$ to $R_{+}(\gamma)$ transversely.

Let $S$ be a compact oriented surface with components $S_{1}, \ldots, S_{n}$. Let $\chi\left(S_{i}\right)$ be the Euler characteristic of $S_{i}$. The Thurston norm of $S$ is defined to be

$$
x(S)=\sum_{\chi\left(S_{i}\right)<0}\left|\chi\left(S_{i}\right)\right|
$$


A sutured manifold $(M, \gamma)$ is taut if

(1) $M$ is irreducible.

(2) $R(\gamma)$ is Thurston norm minimizing in $H_{2}(M, \gamma)$; that is, if $S$ is any other properly embedded surface with $[S]=[R(\gamma)]$, then $x(R(\gamma)) \leq$ $x(S)$.

(3) $R(\gamma)$ is incompressible in $M$.

The following is due to Gabai [7] and Thurston [19].

Theorem 2.1. A sutured manifold $(M, \gamma)$ is taut if and only if it carries a transversely oriented, taut, codimension-1 foliation $\mathcal{F}$.

We require the following result due to Honda et al. $[\mathbf{1 6}]$.

Theorem 2.2. Let $(M, \gamma)$ be an irreducible sutured manifold with annular sutures, and let $(M, \Gamma)$ be the associated convex structure. The following are equivalent:

(1) $(M, \gamma)$ is taut.

(2) $(M, \gamma)$ carries a taut foliation.

(3) $(M, \Gamma)$ carries a universally tight contact strucuture.

(4) $(M, \Gamma)$ carries a tight contact structure.

\section{The end of an open contact manifold and some invariants}

Let $(V, \xi)$ be any open contact 3-manifold which is the interior of a compact 3-manifold $M$ such that $\partial M$ is nonempty and contains at least one component of nonzero genus. Fix an embedding of $V \hookrightarrow \operatorname{int}(M)$ so that we can think of $V$ as $M \backslash \partial M$. Choose a boundary component $S \subset \partial M$ and let $\Sigma \subset M \backslash \partial M$ be an embedded surface isotopic to $S$ in $M$. Note that $S$ and $\Sigma$ bound a contact manifold $(\Sigma \times(0,1), \xi)$. We call such a manifold, along with the embedding into $V$, a contact end corresponding to $S$ and $\xi$. Let Ends $(V, \xi ; S)$ be the collection of contact ends corresponding to $S$ and $\xi$.

Let $S \subset \partial M$ be a component of nonzero genus and let $\lambda \subset S$ be a separating, simple, closed curve which bounds a punctured torus $T$ in $S$. Fix a basis $B$ of the first homology of $T$. Let $\Sigma \subset V$ be a convex surface which is isotopic to $S$ in $M$ and contains a simple, closed curve $\gamma$ with the following properties:

(1) $\gamma$ is isotopic to $\lambda$ on $\Sigma$, where we have identified $\Sigma$ and $S$ by an isotopy in $M$.

(2) $\gamma$ intersects $\Gamma_{\Sigma}$ transversely in exactly two points.

(3) $\gamma$ has minimal geometric intersection number with $\Gamma_{\Sigma}$.

Call any such surface well-behaved with respect to $S$ and $\lambda$. Note that there exists a simple, closed curve $\mu \subset \Gamma_{\Sigma}$ which is contained entirely in $T$. Let the slope of $\Sigma$, written slope $(\Sigma)$, be the slope of $\mu$ measured with respect 
to the basis $B$ of the first homology of $T$. When $S$ is a torus, we omit all references to the curve $\lambda$ as it is unnecessary for our definition.

Let $E \in \operatorname{Ends}(V, \xi ; S)$. Let $\mathcal{C}(E)$ be the set of all well-behaved convex surfaces in the contact end $E$. If $\mathcal{C}(E) \neq \emptyset$, then define the slope of $E$ to be

$$
\operatorname{slope}(E)=\sup _{\Sigma \in \mathcal{C}(E)}(\operatorname{slope}(\Sigma)) \text {. }
$$

Here we allow sup to take values in $\mathbb{R} \cup\{\infty\}$. We topologize $\mathbb{R} \cup\{\infty\}$ as the unit circle. Note that $\operatorname{Ends}(V, \xi ; S)$ is a directed set, directed by reverse inclusion, and that the function slope: $\operatorname{Ends}(V, \xi ; S) \rightarrow \mathbb{R} \cup\{\infty\}$ is a net. If $\mathcal{C}(E)$ is nonempty for a cofinal sequence of contact ends and this net is convergent, then we call the limit the slope at infinity of $(V, \xi ; S, \lambda, B)$ or the slope at infinity of $(V, \xi)$ if $S, \lambda$, and $B$ are understood from the context. If the slope at infinity exists, then we say that this slope is attained if for each $E \in \operatorname{Ends}(V, \xi ; S)$, there exists a $\Sigma \in \mathcal{C}(E)$ with that slope. Note that any slope that is attained must necessarily be rational.

Let $\Sigma \in \mathcal{C}(E)$. Define the division number of $\Sigma$, written $\operatorname{div}(\Sigma)$, to be half the number of dividing curves and $\operatorname{arcs}$ on $T$. When $\Sigma$ is a torus, this is the usual torus division number. If $\mathcal{C}(E) \neq \emptyset$, then let

$$
\operatorname{div}(E)=\min _{\Sigma \in \mathcal{C}(E)}(\operatorname{div}(\Sigma))
$$

Note that div: Ends $(V, \xi, S) \rightarrow \mathbb{N} \cup\{\infty\}$ is a net, where we endow $\mathbb{N} \cup$ $\{\infty\}$ with the discrete topology. If $\mathcal{C}(E)$ is nonempty for a cofinal sequence of contact ends, then we call the limit the division number at infinity of $(V, \xi ; S, \lambda, B)$ or the division number at infinity of $(V, \xi)$ if $S, \lambda$, and $B$ are understood from the context. Note that the slope at infinity and the division number at infinity are proper isotopy invariants.

\section{Classification theorems for tight toric ends}

In this section, we study tight contact structures on toric ends. We say that a toric end is minimally twisting if it contains only minimally twisting toric annuli. We first show that it is possible to refer to the slope at infinity and the division number at infinity for toric ends.

Proposition 4.1. Let $T^{2} \times[0, \infty)$ be a tight toric end. Then the division number at infinity and the slope at infinity are defined.

Proof. First note that $\mathcal{C}(E)$ is nonempty for any end $E$ since the condition for being well-behaved is vacuously true for tori. Also, note that the division number at infinity exists by definition.

If there exists a nested sequence of ends $E_{i}$ such that slope $\left(E_{i}\right)=\infty$, then the slope at infinity is $\infty$. Otherwise, there exists an end $E=T^{2} \times[0, \infty)$ such that for no end $F \subset E$ is $\operatorname{slope}(F)=\infty$. This means that $E$ is minimally twisting. Without loss of generality, assume $T_{i}=T^{2} \times i$ is convex 
with slope $s_{i}$. Note that the $s_{i}$ form a clockwise sequence on the Farey graph and are contained in a half-open arc which does not contain $\infty$. Since slope $(F) \leq s_{i}$ for any end $F \subset T^{2} \times[i, \infty)$, our net is convergent, so the slope at infinity is defined.

4.1. Tight, minimally twisting toric ends with irrational slope at infinity. In this section, we study tight, minimally twisting toric ends $\left(T^{2} \times[0, \infty), \xi\right)$ with irrational slope $r$ at infinity and with convex boundary satisfying $\operatorname{div}\left(T^{2} \times 0\right)=1$ and $\operatorname{slope}\left(T^{2} \times 0\right)=-1$. Unless otherwise specified, all toric ends will be of this type.

We first show how to associate to any such toric end a function $f_{\xi}: \mathbb{N} \rightarrow$ $\mathbb{N} \cup\{0\}$. There exists a sequence of rational numbers $q_{i}$ on the Farey graph which satisfies the following:

(1) $q_{1}=-1$ and the $q_{i}$ proceed in a clockwise fashion on the Farey graph.

(2) $q_{i}$ is connected to $q_{i+1}$ by an arc of the graph.

(3) The $q_{i}$ converge to $r$.

(4) The sequence is minimal in the sense that $q_{i}$ and $q_{j}$ are not joined by an arc of the graph unless $j$ is adjacent to $i$.

We can form this sequence inductively by taking $q_{2}$ to be the rational number which is closest to $r$ on the clockwise arc of the Farey graph $[-1, r]$ between -1 and $r$ and has an edge of the graph from -1 to $q_{2}$. Similarly, construct the remaining $q_{i}$. Any such sequence can be grouped into continued fraction blocks. We say that $q_{i}, \ldots, q_{j}$ form a continued fraction block if there is an element of $S L_{2}(\mathbb{Z})$ taking the sequence to $-1, \ldots,-m$. We call $m$ the length of the continued fraction block. We say that this block is maximal if it cannot be extended to a longer continued fraction block in the sequence $q_{i}$. Since $r$ is irrational, maximal continued fraction blocks exist. Denote these blocks by $B_{i}$. To apply this to our situation, we need the following.

Proposition 4.2. There exists a nested sequence of convex tori $T_{i}$ with $\operatorname{div}\left(T_{i}\right)=1$ such that $\operatorname{slope}\left(T_{i}\right)=q_{i}$. Moreover, any such sequence must leave every compact set.

Proof. By the definition of slope at infinity, for any $\epsilon$, there is an end $E$ such that slope $(E)$ is within $\epsilon$ of $r$. This means that there is a convex torus $T$ in $E$ with slope lying within $2 \epsilon$ of $r$. Note that since our toric end is minimally twisting and has slope $r$ at infinity, slope $(T) \in[-1, r)$. We attach bypasses to $T$ so that $\operatorname{div}(T)=1$. The toric annulus bounded by $T^{2} \times 0$ and $T$ contains the tori $T_{i}$ with $q_{i}$ lying counterclockwise to slope $(T)$. Fix these first $T_{i}$. Choose another torus $T^{\prime}$ outside of the toric annulus with slope even closer to $r$. Again, adjust the division number of $T^{\prime}$ so that it is 1 and factor the toric annulus bounded by $T$ and $T^{\prime}$ to find another finite number of our $T_{i}$. Proceeding in this fashion, we see we have the desired sequence 
of $T_{i}$. Any such sequence must leave every compact set by the definition of the slope at infinity. For, if there were such a sequence of tori, then we could find a torus $T$ in any end with slope $(T)>r$, which would show that the slope at infinity is not $r$.

This factors the toric end according to our sequence of rationals. We say that a consecutive sequence of $T_{i}$ forms a continued fraction block if the corresponding sequence of rationals do. Each maximal continued fraction block $B_{i}$ determines a maximal continued fraction block of tori which we also call $B_{i}$. We think of $B_{i}$ as a toric annulus.

To each continued fraction block, we let $n_{j}$ be the number of positive basic slices in the factorization of $B_{i}$ by $T_{j}$. Define $f_{\xi}: \mathbb{N} \rightarrow \mathbb{N} \cup\{0\}$ by $f_{\xi}(j)=n_{j}$. To show that the function $f_{\xi}$ is independent of the factorization by $T_{i}$, suppose $T_{i}^{\prime}$ is another factorization with the same properties as $T_{i}$. Let $B_{j}^{\prime}$ denote the corresponding continued fraction blocks. Fix $j$. There exists $n$ large such that the toric annulus $A$ bounded by $T_{n}$ and $T_{1}$ contains the continued fraction blocks $B_{j}$ and $B_{j}^{\prime}$. Extend the partial factorization of $A$ by $B_{j}^{\prime}$. Recall that one can compute the relative Euler class via such a factorization and that it depends on the number of positive basic slices in each continued fraction block $[\mathbf{1 3}]$. Therefore, $B_{j}$ and $B_{j}^{\prime}$ must have the same number of positive basic slices.

Given an irrational number $r$, let $\mathcal{F}(r)$ denote the collection of functions $f: \mathbb{N} \rightarrow \mathbb{N} \cup\{0\}$ such that $f(i)$ does not exceed one less than the length of $B_{i}$. We can now state a complete classification of the toric ends under consideration.

Theorem 4.3. Let $\left(T^{2} \times[0, \infty), \xi\right)$ be a tight, minimally twisting toric end with convex boundary satisfying $\operatorname{div}\left(T^{2} \times 0\right)=1$ and $\operatorname{slope}\left(T^{2} \times 0\right)=-1$. Suppose that the slope at infinity is irrational. To each such tight contact structure, we can assign a function $f_{\xi}: \mathbb{N} \rightarrow \mathbb{N} \cup\{0\}$ which is a complete proper isotopy (relative to the boundary) invariant. Moreover, given any $f \in \mathcal{F}(r)$, there exists a toric end $\left(T^{2} \times[0, \infty), \xi\right)$ such that $f_{\xi}=f$.

Proof. If $f_{\xi}=f_{\xi^{\prime}}$, then we can shuffle bypasses within any given continued fraction block so that all positive basic slices occur at the beginning of the block. Since the number of positive basic slices in any continued fraction block is the same, it is clear that they are properly isotopic.

It is a straightforward application of the gluing theorem for basic slices in $[\mathbf{1 3}]$ to show that we can construct a toric annulus corresponding to the desired continued fraction blocks. The fact that they stay tight under gluing follows from the fact that overtwisted disks are compact.

Corollary 4.4. Let $\left(T^{2} \times[0,1), \xi\right)$ be a tight, minimally twisting toric end with irrational slope $r$ at infinity and $\operatorname{slope}\left(T^{2} \times\{0\}\right)=-1$. Suppose that for 
each $i$, there exist $j, k \geq i$ such that $f_{\xi}(j)>0$ and $f_{\xi}(k)=0$. Then there does not exist any tight, toric end $\left(T^{2} \times[0, \infty), \eta\right)$ such that $\left.\xi\right|_{T^{2} \times[0,1)}=\left.\eta\right|_{T^{2} \times[0,1)}$.

Proof. For contradiction, assume that there was an inclusion $\phi:\left(T^{2} \times\right.$ $[0,1), \xi) \rightarrow\left(T^{2} \times[0, \infty), \eta\right)$. Perturb $T^{2} \times\{2\}$ to be convex of slope $b$. Without loss of generality, we can assume that the toric annulus bounded by $T^{2} \times\{0\}$ and $T^{2} \times\{2\}$ is minimally twisting. We have a minimal, clockwise sequence of rationals $q_{l}$ for $1 \leq l \leq n$ on the Farey graph such that $q_{1}=-1, q_{n}=b$, and $q_{l}$ is joined to $q_{l+1}$ by an arc of the Farey graph. There exists $m \leq n-1$ such that $r$ lies on the clockwise arc between $q_{m}$ and $q_{m+1}$. By the definition of the slope at infinity, there exists a torus $T_{\mathrm{in}} \subset T^{2} \times[0,1)$ with slope $\left(T_{\mathrm{in}}\right)=q_{m}$. By the hypothesis on $f_{\xi}$, there exist two continued fraction blocks, $B_{1}$ and $B_{2}$, contained in $T^{2} \times[0,1)$ and lying outside the toric annulus bounded by $T^{2} \times\{0\}$ and $T_{\text {in }}$, with the following property: $B_{1}$ has a negative basic slice and $B_{2}$ has a positive basic slice. This follows from the definition of the function $f_{\xi}$. Choose a torus $T^{\prime}$ so that the toric annulus bounded by $T_{\text {in }}$ and $T^{\prime}$ contains $B_{1}$ and $B_{2}$. Let $T_{\text {out }}$ be a torus with slope $q_{m+1}$ that lies between $T^{\prime}$ and $T^{2} \times\{2\}$. Observe that $T_{\text {in }}$ and $T_{\text {out }}$ bound a basic slice. But, this basic slice was formed by gluing basic slices of opposite signs, since $B_{1}$ and $B_{2}$ are contained in this basic slice. This contradicts the tightness of the contact structure $\eta$ by $[\mathbf{1 3}]$.

4.2. Tight, minimally twisting toric ends with rational slope at infinity. We now consider tight, minimally twisting toric ends $\left(T^{2} \times\right.$ $[0, \infty), \xi)$ with rational slope $r$ at infinity and with convex boundary satisfying $\operatorname{div}\left(T^{2} \times 0\right)=1$ and $\operatorname{slope}\left(T^{2} \times 0\right)=-1$. Unless otherwise specified, all toric ends will be of this type. We first deal with the situation when the slope at infinity is not attained.

We show how to every toric end under consideration we can assign a function

$$
f_{\xi}:\{1, \ldots, n(r)\} \times\{1,-1\} \rightarrow \mathbb{N} \cup\{0, \infty\} .
$$

We proceed in a fashion similar to the irrational case. Given $r$ rational, there exists a sequence of rationals $q_{i}$ satisfying the following:

(1) $q_{1}=-1$ and $q_{i}$ proceed in a clockwise fashion on the Farey graph.

(2) $q_{i}$ are connected to $q_{i+1}$ by an arc of the tesselation.

(3) $q_{i}$ converge to $r$, but $q_{i} \neq r$ for any $i$.

(4) The sequence is minimal in the sense that $q_{i}$ and $q_{j}$ are not joined by an arc of the tesselation unless $j$ is adjacent to $i$.

We construct such a sequence inductively just as in the irrational case, except we never allow the rationals $q_{i}$ to reach $r$. Note that such a sequence breaks up naturally into $n-1$ finite continued fraction blocks $B_{i}$ and one infinite continued fraction block $B_{n}$ (i.e., $B_{n}$ can be taken to the negative integers after action by $\left.S L_{2}(\mathbb{Z})\right)$. Note that $n$ is completely determined 
by $r$. Just as in the irrational case, there exist nested convex tori $T_{i}$ with $\operatorname{div}\left(T_{i}\right)=1$ and $\operatorname{slope}\left(T_{i}\right)=q_{i}$. We can argue as in the irrational case to show that these tori must leave every compact set of the toric end. We will also refer to the collection of tori $T_{i}$ corresponding to $B_{i}$ by the same name.

We will now construct $f_{\xi}$. Let $f_{\xi}(i, \pm 1$ ) be the number of positive (negative) basic slices in the continued fraction block $B_{i}$. Of course, for a finite continued fraction block, $f_{\xi}(i, 1)$ determines $f_{\xi}(i,-1)$. However, this is clearly not the case for $B_{n}$.

As in the irrational case, let $\mathcal{F}(r)$ be the collection of functions $f:\{1, \ldots$, $n(r)\} \times\{1,-1\} \rightarrow \mathbb{N} \cup\{0, \infty\}$ such that $f_{\xi}(i, 1)+f_{\xi}(i,-1)=\left|B_{i}\right|-1$ for $i \leq n-1$, where $\left|B_{i}\right|$ is the length of $B_{i}$, and at least one of $f_{\xi}(n(r), \pm 1)$ is infinite. Recall that $\left|B_{i}\right| \geq 2$.

Theorem 4.5. Let $\left(T^{2} \times[0, \infty), \xi\right)$ be a tight, minimally twisting toric end with convex boundary satisfying $\operatorname{div}\left(T^{2} \times 0\right)=1$ and $\operatorname{slope}\left(T^{2} \times 0\right)=-1$. Suppose that the slope at inifinity is rational and is not attained. To each such tight contact structure, we can assign a function $f_{\xi}:\{1, \ldots, n(r)\} \times$ $\{1,-1\} \rightarrow \mathbb{N} \cup\{0, \infty\}$ which is a complete proper isotopy (relative to the boundary) invariant. Moreover, for any $f \in \mathcal{F}(r)$, there exists a tight, minimally twisting toric end $\left(T^{2} \times[0, \infty), \xi\right)$ with slope $r$ at infinity which is not realized such that $f=f_{\xi}$.

Proof. Suppose $f_{\xi}=f_{\xi}^{\prime}$. As in the irrational case, we can adjust our factorization of the finite continued fraction blocks so that all of the positive basic slices occur first in each continued fraction block. Therefore, we can isotope the two contact structures so that they agree on the first $n-1$ continued fraction blocks.

We now consider the infinite basic slice. Without loss of generality, we may assume that the infinite basic slices for $\xi$ and $\xi^{\prime}$ are toric ends $\left(T^{2} \times\right.$ $[0, \infty), \xi)$ and $\left(T^{2} \times[0, \infty), \xi^{\prime}\right)$ with slope $\left(T^{2} \times\{0\}\right), \operatorname{div}\left(T^{2} \times\{0\}\right)$, and infinite slope at infinity that is not realized. The corresponding factorization is then given by nested tori $T_{i}$ and $T_{i}^{\prime}$ such that $\operatorname{slope}\left(T_{i}\right)=\operatorname{slope}\left(T_{i}^{\prime}\right)=-i$ and $\operatorname{div}\left(T_{i}\right)=1$. We now construct model toric ends $\xi_{n}^{ \pm}$and $\xi_{\text {alt }}$ and show that any infinite basic slice is properly isotopic to one of the models. Let $B_{i}^{ \pm}$be the positive (negative) basic slice with slope $\left(T^{2} \times 0\right)=-i$ and slope $\left(T^{2} \times 1\right)=$ $-i-1$. Let $\xi_{n}^{ \pm}$be the toric end constructed as $B_{1}^{ \pm} \cup \cdots \cup B_{n}^{ \pm} \cup B_{n+1}^{\mp} \cup \cdots$. Let $\xi_{\text {alt }}$ be $B_{1}^{+} \cup B_{2}^{-} \cup B_{3}^{+} \cup \cdots$. First consider the case when $f_{\xi}(n, 1)=m$. There exists $N$ large so that the toric annulus bounded by $T_{1}$ and $T_{N}$ contains at least $m$ positive basic slices and $m$ negative basic slices. By shuffling bypasses in this toric annulus, we can rechoose our factorization so that all positive bypass layers occur first in our factorization. This toric end is clearly properly isotopic to $\xi_{m}^{+}$. We handle the case when $f_{\xi}(n,-1)=m$ similarly. Now, suppose that $f_{\xi}(n, \pm 1)=\infty$. Fix some number $k$. Choose $N_{1}$ large enough that the toric annulus bounded by $T_{1}$ and $T_{N_{1}}$ contains at 
least $k$ positive and $k$ negative basic slices. By shuffling bypasses in this toric annulus, we can arrange for the first $2 k$ basic slices in the factorization to be alternating. There exists an isotopy $\phi_{t}^{1}$ such that $\phi_{0}^{1}$ is the identity and $\phi_{1 *}^{1}(\xi)$ agrees with $\xi_{\text {alt }}$ in the first $2 k$ basic slices. Call the pushed forward contact structure by the same name. There exists $N_{2}$ large such that $T_{2 k}$ and $T_{N_{2}}$ bound a toric annulus with $k$ positive and $k$ negative basic slices. Leaving the first $2 k$ tori in our factorization fixed, we can shuffle bypasses in the toric annulus bounded by $T_{2 k}$ and $T_{N_{2}}$ so that signs are alternating. Choose an isotopy $\phi_{t}^{2}$ as before such that $\phi_{t}^{2}$ is the identity on the toric annulus bounded by $T_{1}$ and $T_{2 k}$ and takes the second $2 k$ basic slices of $\xi$ onto those of $\xi_{\text {alt }}$. Continuing in this fashion, we can construct $\phi_{t}^{n}$, which is supported on $K_{n}$ compact such that $K_{i} \subset K_{i+1}$ and $T^{2} \times[0, \infty)=\cup K_{i}$. Hence we have an isotopy taking $\xi$ to $\xi_{\text {alt }}$. The existence result follows immediately from Honda's gluing results for toric annuli [13].

Corollary 4.6. Let $\left(T^{2} \times[0,1), \xi\right)$ be a tight, minimally twisting toric end that does not attain a rational slope $r$ at infinity. Suppose $f_{\xi}(n(r) \times\{1\})$ and $f_{\xi}(n(r) \times\{-1\})$ are nonzero. Then there does not exist any tight, toric end $\left(T^{2} \times[0, \infty), \eta\right)$ such that $\left.\xi\right|_{T^{2} \times[0,1)}=\left.\eta\right|_{T^{2} \times[0,1)}$.

Proof. Assume that there was such an inclusion $\phi:\left(T^{2} \times[0,1), \xi\right) \rightarrow$ $\left(T^{2} \times[0, \infty), \eta\right)$. Let $T_{i}$ be the first torus in the factorization of the infinite continued fraction block of $\left(T^{2} \times[0,1), \xi\right)$. By definition, there exists another torus $T_{j}$ with $j>i$ such that $T_{i}$ and $T_{j}$ bound basic slices of both signs. By the definition of the slope at infinity, there exists a convex torus $T$ outside of the toric annulus bounded by $\phi\left(T_{i}\right)$ and $\phi\left(T_{j}\right)$ which has slope $r$. Note that $\phi\left(T_{i}\right)$ and $T$ bound a basic slice which is formed by gluing basic slices of opposite signs. This implies that $\left(T^{2} \times[0, \infty), \eta\right)$ is overtwisted $[\mathbf{1 3}]$.

Corollary 4.4 and Corollary 4.6 will be essential to prove Theorem 1.1. We now consider tight, minimally twisting toric ends that realize the slope at infinity and have finite division number at infinity.

Theorem 4.7. Tight, minimally twisting toric ends with finite division number $d$ at infinity that realize the slope $r$ at infinity are in one-to-one correspondence with tight, minimally twisting contact structures on $T^{2} \times[0,1]$ with $T^{2} \times i$ convex, $\operatorname{slope}\left(T^{2} \times 0\right)=-1, \operatorname{slope}\left(T^{2} \times 1\right)=r, \operatorname{div}\left(T^{2} \times 0\right)=1$, and $\operatorname{div}\left(T^{2} \times 1\right)=d$ up to isotopy relative to $T^{2} \times 0$.

Proof. Let $\left(T^{2} \times[0, \infty), \xi\right)$ be such a toric end. By the definition of division number at infinity and slope at infinity, there exists a convex torus $T$ with the following properties:

(1) $\operatorname{div}(T)=d$

(2) $\operatorname{slope}(T)=r$

(3) Any other convex torus $T^{\prime}$ lying in the noncompact component of $T^{2} \times[0, \infty) \backslash T$ satisfies $\operatorname{div}\left(T^{\prime}\right) \geq d$. 
Any such torus will necessarily have slope $r$. Let $A$ be the toric annulus bounded by $T^{2} \times 0$ and $T$. We know that any other torus $T^{\prime}$ with the same properties as $T$ bounds a toric annulus $A^{\prime}$ that is topologically isotopic to $A$. By the definition of $T$ and $T^{\prime}$, there exists a torus $T^{\prime \prime}$ outside of $A$ and $A^{\prime}$ that has the same properties as $T$. Since $\xi$ is minimally twisting, $T^{\prime}$ and $T^{\prime \prime}$ bound a vertically invariant toric annulus. Similarly, $T$ and $T^{\prime \prime}$ bound a vertically invariant toric annulus. We can use these toric annuli to isotope $A$ and $A^{\prime}$ to the same toric annulus in our toric end. This yields the desired correspondence. Given a tight, minimally twisting contact structures on $T^{2} \times[0,1]$ with $T^{2} \times i$ convex, $\operatorname{slope}\left(T^{2} \times 0\right)=-1, \operatorname{slope}\left(T^{2} \times 1\right)=r$, $\operatorname{div}\left(T^{2} \times 0\right)=1$, and $\operatorname{div}\left(T^{2} \times 1\right)=d$, we obtain a toric end by removing $T^{2} \times 1$.

Let $A_{1}=S^{1} \times[0,1]$ and $A_{2}=S^{1} \times[0,1]$ be convex annuli with Legendrian boundary such that $t b\left(S^{1} \times 0\right)=-1$ and $t b\left(S^{1} \times 1\right)=-m$. We say that $A_{1}$ and $A_{2}$ are stabily disk equivalent if there exist disk equivalent convex annuli $A_{i}^{\prime}=S^{1} \times[0,2]$ such that $t b\left(S^{1} \times 1\right)=-1, t b\left(S^{1} \times 2\right)=-n<-m$, and $A_{i}=S^{1} \times[0,1] \subset A_{i}^{\prime}$.

Theorem 4.8. Let $\left(T^{2} \times[0, \infty), \xi\right)$ be a tight, minimally twisting toric end with slope $\left(T^{2} \times 0\right)=\infty$, slope $\infty$ at infinity, and division number $\infty$ at infinity. Then we can associate to $\xi$ a collection of nested families of convex annuli $A_{i}=S^{1} \times[0, i]$ with Legendrian boundary such that $t b\left(S^{1} \times 0\right)=-1$, $t b\left(S^{1} \times i+1\right)=t b\left(S^{1} \times i\right)+1$ such that any two annuli $A_{i}$ and $A_{i}^{\prime}$ in different families are stabily disk equivalent.

Proof. To construct such annuli, simply choose a factorization of the toric end by tori $T_{i}$ such that $T_{1}=T^{2} \times 0, \operatorname{slope}\left(T_{i}\right)=\infty, \operatorname{div}\left(T_{i+1}\right)=\operatorname{div}\left(T_{i}\right)+1$, and the $T_{i}$ leave every compact set. Let $A_{1}$ be the convex annulus with boundary on $T_{1}$ and $T_{2}$. Choose $A_{1}^{\prime}$, a horizontal convex annulus between $T_{2}$ and $T_{3}$ which shares a boundary component with $A_{1}$. Let $A_{2}=A_{1} \cup A_{1}^{\prime}$. Continuing in this fashion, we construct a sequence of nested annuli $A_{i}$. Now, choose any other factorization by tori $T_{i}^{\prime}$ satisfying the same properties as the $T_{i}$ and let $A_{i}^{\prime}$ be the corresponding sequence of convex annuli. We will show that $A_{i}$ is stabily disk equivalent to $A_{i}^{\prime}$. Choose $N$ large so that the toric annulus bounded by $T_{1}$ and $T_{N}$ contains $A_{i}$ and $A_{i}^{\prime}$. Let $A$ be a convex annulus between the $S^{1} \times i \subset A_{i}^{\prime}$ and a horizontal Legendrian curve on $T_{N}$. Let $A^{\prime}=A_{i}^{\prime} \cup A$. Honda's result in [13] implies that $A$ and $A^{\prime}$ are disk equivalent.

Corollary 4.9. Any tight, minimally twisting toric end $\left(T^{2} \times[0, \infty), \xi\right)$ with slope $\left(T^{2} \times 0\right)=\infty$, slope $\infty$ at infinity, and division number $\infty$ at infinity embeds in a vertically invariant neighborhood of $T^{2} \times 0$.

Proof. Honda's model [13] for increasing the torus division number can be applied inductively on a vertically invariant neighborhood of $T^{2} \times 0$ to create the desired sequence of nested tori $T_{i}$ and corresponding annuli $A_{i}$. 
The contact structure on the toric annulus bounded by $T_{1}$ and $T_{i}$ is uniquely determined by $A_{i}[\mathbf{1 3}]$.

We are lead to the following question:

Question 4.10. What are necessary and sufficient conditions for two toric ends with infinite division number at infinity to be properly isotopic?

4.3. Nonminimally twisting, tight toric ends. In this section, we deal with tight toric ends $\left(T^{2} \times[0, \infty), \xi\right)$, with slope $\left(T^{2} \times 0\right)=0$ and $\operatorname{div}\left(T^{2} \times 0\right)=$ 1 , that are not minimally twisting. We first recall Honda's classification for nonminimally twisting tight contact structures on $T^{2} \times[0,1]$ in $[\mathbf{1 3}]$. He constructs a family $\xi_{n}^{ \pm}$of tight, rotative contact structures on $T^{2} \times[0,1]$ with slope $\left(T^{2} \times i\right)=0$ and $\operatorname{div}\left(T^{2} \times i\right)=1$ and shows that this is a complete and nonoverlapping list of contact structures satisfying these conditions. We define the rotativity of a tight toric end $\xi$ with slope $\left(T^{2} \times 0\right)=0$ and $\operatorname{div}\left(T^{2} \times 0\right)=1$ to be the maximum $n$ such that there is an embedding $e:\left(T^{2} \times[0,1], \xi_{n}^{ \pm}\right) \hookrightarrow\left(T^{2} \times[0, \infty), \xi\right)$ with $e\left(T^{2} \times 0\right)=T^{2} \times 0$. If no maximum exists, then we say that $\xi$ has infinite rotativity. If $n$ is the rotativity of $\xi$, then $\xi_{n}^{+}$and $\xi_{n}^{-}$cannot both be embedded in $\xi$. Assume for contradiction that there are two such embeddings $e_{+}$and $e_{-}$, respectively. Then the images of these two embeddings are contained in a common toric annulus $T^{2} \times[0,1]$, where $\operatorname{div}\left(T^{2} \times\{1\}\right) \geq 1$. If $\operatorname{div}\left(T^{2} \times\{0\}\right)=1$, then it follows from the factorization theorems concerning such toric annuli in [13] that these embeddings cannot coexist. If $\operatorname{div}\left(T^{2} \times\{0\}\right)>1$, then the closure of the exterior of the image of $e_{+}$inside $T^{2} \times[0,1]$ is a nonrotative outer layer (similarly for $e_{-}$). By Honda's work in [13], the images of $e_{+}$ and $e_{-}$must therefore have the same sign. But, this contradicts the very existence of the two different embeddings $e_{+}$and $e_{-}$. Hence, we can refer to the sign of rotativity as well. We construct two more nonminimally twisting toric ends $\xi_{\infty}^{ \pm}$. Set $\left(T^{2} \times[0, \infty), \xi_{\infty}^{ \pm}\right)=\cup_{i=1}^{\infty}\left(T^{2} \times[0,1], \xi_{2}^{ \pm}\right)$.

Theorem 4.11. Let $\left(T^{2} \times[0, \infty), \xi\right)$ be a tight toric end that is not minimally twisting and such that $\operatorname{slope}\left(T^{2} \times\{0\}\right)=0$ and $\operatorname{div}\left(T^{2} \times\{0\}\right)=1$.

(1) Assume that $\xi$ has finite rotativity and that the slope at infinity is $s$ and is not attained. Then $\xi$ is uniquely determined by $n$ and the sign of rotativity. Moreover, $\xi$ is universally tight.

(2) Assume that $\xi$ has finite rotativity, the slope at infinity is $s$ and is attained, and the division number at infinity is $k<\infty$. Such $\xi$ are in one-to-one correspondence with tight, toric annuli $T^{2} \times[0,1]$ with $\operatorname{slope}\left(T^{2} \times\{0\}\right)=0, \operatorname{slope}\left(T^{2} \times\{1\}\right)=s, \operatorname{div}\left(T^{2} \times\{0\}\right)=1$, and $\operatorname{div}\left(T^{2} \times\{1\}\right)=k$, up to isotopy relative to $T^{2} \times\{0\}$. Moreover, all such $\xi$ are universally tight.

(3) Assume that $\xi$ has slope $s$ at infinity and infinite division number at infinity (the rotativity must necessarily be finite). We can factor $\xi$ into 
a toric annulus $T^{2} \times[0,1]$ with $\operatorname{slope}\left(T^{2} \times\{1\}\right)=s$ and $\operatorname{div}\left(T^{2} \times\{1\}\right)=$ 1 and a minimally twisting, toric end $T^{2} \times[1, \infty)$. Moreover, the contact structure on the toric annulus is uniquely determined by $\xi$, and $\xi$ is universally tight. To the toric end $T^{2} \times[1, \infty)$, we can assign a family of annuli $A_{i}$ as in Theorem 4.8 that is unique up to stable disk equivalence.

(4) Assume that $\xi$ has infinite rotativity. Then $\xi$ is properly isotopic relative to the boundary to either $\xi_{\infty}^{+}$or $\xi_{\infty}^{-}$, so the sign of rotativity is defined in the infinite case as well. Moreover, the $\xi_{\infty}^{ \pm}$are universally tight.

Proof. First, consider the case of finite rotativity when the slope at infinity is not attained. Assume that the sign of rotativity is + . Factor off a toric annulus $\xi_{n}^{+}$. What remains is a minimally twisting, toric end. Based on previous classification results for these ends, it suffices to determine the number of positive basic slices in each continued fraction block. Note that the sign of the basic slices in the continued fraction blocks is determined by the sign of rotativity, just as in [13]. Since all basic slices have the same sign, $\xi$ is universally tight just as in $[\mathbf{1 3}]$. The proof of the case when the slope at infinity is attained and the division number at infinity is finite is essentially identical to the analogous case when the toric end is minimally twisting.

The case of infinite division number at infinity is similar to previous cases. We first show that the toric annulus in the factorization is unique. Choose two such factorizations by tori $T$ and $T^{\prime}$. These tori are contained in a larger toric annulus $T^{2} \times[0,2] . T$ and $T^{2} \times\{2\}$ and $T^{\prime}$ and $T^{2} \times\{2\}$ bound nonrotative outer layers. By [13], we know that the toric annuli bounded by $T$ and $T^{2} \times\{0\}$ and $T^{\prime}$ and $T^{2} \times\{0\}$ must therefore be the same. The fact that $\xi$ is universally tight is virtually identical to the previous cases. The statement concerning the minimally twisting toric annulus follows from the proof of Theorem 4.8 .

Now, assume $\xi$ has infinite rotativity. First, note that we cannot have two embeddings $e_{n}^{ \pm}:\left(T^{2} \times[0,1], \xi_{n}^{ \pm}\right) \hookrightarrow\left(T^{2} \times[0, \infty), \xi\right)$ with $e_{n}^{ \pm}\left(T^{2} \times 0\right)=T^{2} \times 0$ as previously discussed. Since $\xi$ has infinite rotativity, there exists a sequence of, say, positive embeddings $e_{n}:\left(T^{2} \times[0, n], \xi_{n}^{+}\right) \hookrightarrow\left(T^{2} \times[0, \infty), \xi\right)$ with $e_{n}\left(T^{2} \times 0\right)=T^{2} \times 0$. Moreover, we can take this sequence of embeddings to be nested in the sense that $e_{n}=e_{n+1}$ on $[0, n]$. This follows immediately by factoring a toric annulus containing the images of $e_{n}$ and $e_{n+1}$. Note that any sequence of such embeddings must necessarily leave any compact set. We can use this sequence of embeddings to construct a proper isotopy of $\xi$ with $\xi_{\infty}^{+}$as in the proof of Theorem 4.5. Again, the fact that $\xi_{\infty}^{ \pm}$ are universally tight follows from the fact that nonminimally twisting toric annuli are universally tight. 
Corollary 4.12. Let $\left(T^{2} \times[0, \infty), \xi\right)$ be a tight toric end that is not minimally twisting and has finite rotativity. Then $\left(T^{2} \times[0, \infty), \xi\right)$ embeds into a toric annulus $\left(T^{2} \times[0,1], \eta\right)$ with convex boundary.

Proof. In the case when the slope at infinity is not attained, the obstruction to finding an embedding, the mixing of signs of basic slices in continued fraction blocks, is not present. Therefore, such embeddings exist and are straightforward to construct using the techniques in $[\mathbf{1 3}]$. In the case when the slope is attained and the division number is finite, the embedding comes for free. When the division number at infinity is infinite, one must use the folding trick in a vertically invariant neighborhood of a convex torus described in [13] and already used in our discussion of minimally twisting toric ends with infinite division number at infinity.

\section{Classifying tight contact structures on $S^{1} \times \mathbb{R}^{2}$ and $T^{2} \times \mathbb{R}$}

We now show that in many cases, the classification of tight contact structures on $S^{1} \times \mathbb{R}^{2}$ and $T^{2} \times \mathbb{R}$ reduces to the classification of toric ends.

5.1. Factoring tight contact structures on $S^{1} \times \mathbb{R}^{2}$. Let $\left(S^{1} \times \mathbb{R}^{2}, \xi\right)$ be a tight contact structure and let $r$ be the slope at infinity. Consider the collection of points on the Farey graph of the form $1 / n$ where $n \in \mathbb{Z}$. Let $s(r)=1 / n$ be the point closest to $r$ (when traversing the Farey graph counterclockwise from $r$ ) that is realized as the slope of a convex torus $T$ topologically isotopic to $S^{1} \times S^{1}$. We can then factor $\left(S^{1} \times \mathbb{R}^{2}, \xi\right)$ into $\left(S^{1} \times D^{2}, \xi\right)$ and $\left(T^{2} \times[0, \infty), \xi\right)$. To see that this factorization is unique, consider any other torus $T^{\prime}$ satisfying the same conditions as $T$. Both $T$ and $T^{\prime}$ lie in a common solid torus $S$ with convex boundary. Note that the toric annuli bounded by $\partial S$ and $T$ and by $\partial S$ and $T^{\prime}$ are identical by the uniqueness of such factorizations on solid tori. This proves the following:

Theorem 5.1. Tight contact structures on $\left(S^{1} \times \mathbb{R}^{2}, \xi\right)$ with nonzero slope at infinity are in one-to-one correspondence with isotopy classes relative to the boundary of tight, minimally twisting toric ends $\left(T^{2} \times[0, \infty), \eta\right)$ with $\operatorname{div}\left(T^{2} \times\{0\}\right)=1$. Tight contact structures on $\left(S^{1} \times \mathbb{R}^{2}, \xi\right)$ with slope zero at infinity are in one-to-one correspondence with isotopy classes relative to the boundary of tight, minimally twisting toric ends $\left(T^{2} \times[0, \infty), \eta\right)$ which do not attain the slope at infinity.

5.2. Factoring tight contact structures on $T^{2} \times \mathbb{R}$. In this section, we deal with tight contact structures on $T^{2} \times \mathbb{R}$. Any convex, incompressible torus $T \subset T^{2} \times \mathbb{R}$ produces a factorization of $T^{2} \times \mathbb{R}$ into $T^{2} \times(-\infty, 0]$ and $T^{2} \times[0, \infty)$. We identify $T^{2} \times(-\infty, 0]$ with $T^{2} \times[0, \infty)$ via reflection about the origin in $\mathbb{R}$ to obtain a negative contact structure on $T^{2} \times[0, \infty)$. We change this to a positive contact structure by reflecting across the $(1,0)$ 
curve in $T^{2}$. Let $\left(T^{2} \times[0, \infty), \xi_{ \pm}\right)$and $\left(T^{2} \times[0, \infty), \xi_{ \pm}^{\prime}\right)$ be two factorizations corresponding to two different convex tori $T$ and $T^{\prime}$ with division number 1 and slope $s$. We see that by keeping track of the $I$-twisting of a toric annulus in $T^{2} \times \mathbb{R}$ containing $T$ and $T^{\prime}$, we can obtain $\left(T^{2} \times[0, \infty), \xi_{ \pm}\right)$from $\left(T^{2} \times[0, \infty), \xi_{ \pm}^{\prime}\right)$ as follows: Remove a (possibly) rotative $T^{2} \times[0,1]$ with $\operatorname{div}\left(T^{2} \times i\right)=1$ and slope $\left(T^{2} \times i\right)=s$ from the boundary of $\left(T^{2} \times[0, \infty), \xi_{+}\right)$ (or $\left(T^{2} \times[0, \infty), \xi_{-}\right)$). Apply a suitable diffeomorphism to $T^{2} \times[0,1]$. Then, glue $T^{2} \times[0,1]$ to the boundary of $\left(T^{2} \times[0, \infty), \xi_{-}\right)\left(\right.$or $\left.\left(T^{2} \times[0, \infty), \xi_{+}\right)\right)$. We call this procedure shifting the rotativity between $\left(T^{2} \times[0, \infty), \xi_{+}\right)$and $\left(T^{2} \times[0, \infty), \xi_{-}\right)$.

Theorem 5.2. Let $\left(T^{2} \times \mathbb{R}, \xi\right)$ be a tight contact manifold which contains a convex, incompressible torus $T$ with $\operatorname{div}(T)=1$ and $\operatorname{slope}(T)=s$. Then the factorization of $\left(T^{2} \times \mathbb{R}, \xi\right)$ into toric ends $\left(T^{2} \times[0, \infty), \xi_{ \pm}\right)$is unique up to shifting the rotativity between the two toric ends.

Theorem 5.2 shows that the classification of contact structures on $T^{2} \times \mathbb{R}$ reduces to the study of toric ends if there is a convex, incompressible torus $T$ with $\operatorname{div}(T)=1$. If $\left(T^{2} \times \mathbb{R}, \xi\right)$ contains no such torus, then the situation is much more subtle.

Question 5.3. If $\left(T^{2} \times \mathbb{R}, \xi\right)$ contains no convex, incompressible torus with division number 1 , then what is the relationship between two factorizations by convex, incompressible tori of minimal torus division number?

\section{Proof of Theorem 1.1}

The proof of Theorem 1.1 is essentially a compilation of the results in previous sections.

We first prove the theorem for $T^{2} \times[0,1)$. Let $r$ be an irrational number. Define the family of functions $f_{n}: \mathbb{N} \rightarrow \mathbb{N} \cup\{0\}$ as follows: $f_{n}(i)>0$ for $i \leq n$ or when $i$ is even, and $f_{n}(i)=0$ when $i>n$ and $i$ is odd. Theorem 4.3 and Corollary 4.6 guarantee that the family of contact structures associated with $f_{n}$ are distinct and not extendible. If $r$ is rational, then define a family of functions $f_{m}:\{1, \ldots, n(r)\} \times\{1,-1\} \rightarrow \mathbb{N} \cup\{0, \infty\}$ as follows: $f_{m}(i \times\{1\})=$ 0 for $i<=n(r)-1, f_{m}(n(r) \times\{1\})=m$, and $f_{m}(n(r) \times\{-1\})=\infty$. By Theorem 4.5 and Corollary 4.6, the family of contact structures associated to $f_{m}$ are distinct and are not extendible.

Theorem 5.1 and Theorem 5.2 now finish the cases of $T^{2} \times \mathbb{R}$ and $S^{1} \times D^{2}$.

\section{Proof of Theorem 1.2 and Theorem 1.3}

Before beginning the proof of Theorem 1.2, we prove a result which allows us to choose the dividing set on $\partial M$ nicely. Let $\Sigma$ be a genus $n$ surface. In Figure 1 , we specify $\alpha_{i}, \beta_{i}$, and $\lambda_{j}$ for a genus 3 surface. For a higher genus $\Sigma$, make the analogous specification. 


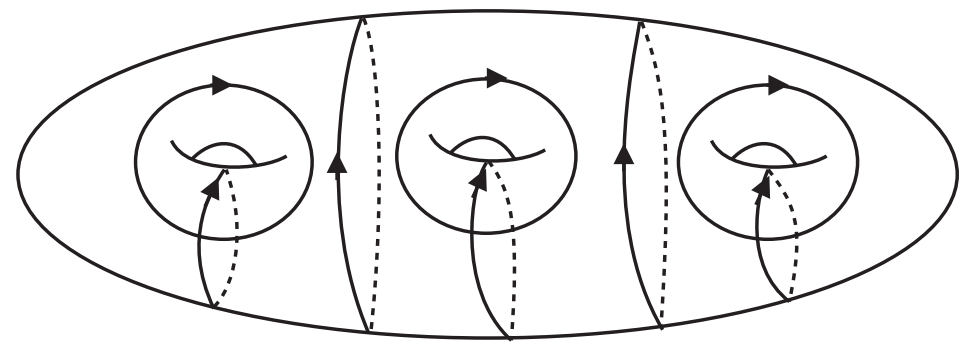

Figure 1. For $1 \leq i \leq 3$, let the $\alpha_{i}$ be the half-hidden, nonseparating, simple, closed curves and let $\beta_{i}$ be the nonseparating curves such that $\alpha_{i} \cdot \beta_{i}=1$ (with subscript increasing from left to right). Let the $\lambda_{j}$ be the two separating curves again labeled left to right.

Lemma 7.1. Let $M$ be any 3-manifold with connected boundary of genus $n$. Let $K$ be the kernel of the map $H_{1}(\partial M ; \mathbb{Q}) \rightarrow H_{1}(M ; \mathbb{Q})$ induced from inclusion. There exists an identification of $\partial M$ with $\Sigma$ such that $\alpha_{i}$ form a basis for $K \subset H_{1}(\partial M ; \mathbb{Q})$ as vector space over $\mathbb{Q}$. Moreover, there exist integers $n_{i}$ and embedded, orientable surfaces $\Sigma_{i}$ such that $\partial \Sigma_{i}$ consists of $n_{i}$ parallel copies of $\alpha_{i}$.

Proof. Let $S_{1}$ be the first cutting surface in a Haken decomposition for $M$. We may assume that no collection of components of $\partial S_{1}$ is separating in $\partial M$ and that $S_{1}$ is orientable [11]. We may also assume that $\partial S_{1}$ consists of parallel copies of a nonseparating, simple, closed curve that we identify with $\alpha_{1}$. If $\partial S_{1}$ is not all parallel, then two boundary components $b_{1}$ and $b_{2}$ can be chosen so that there exists an arc $\mu$ joining the $b_{i}$ that does not intersect any other components of $\partial S_{1}$. Let $A$ be a small annular neighborhood of $\mu$. Since $\partial S_{1}$ is nonseparating, we can choose $\mu$ so that $S_{1} \cup A$ is an oriented surface with $b_{i}$ replaced by a new boundary component homologous to $b_{1}+b_{2}$. We can continue this process until the boundary components of $S_{1}$ consist of $n_{1}$ copies of simple closed curve which we identify with $\alpha_{1}$. Form a new 3-manifold $M_{1}$ by attaching a 2-handle $H_{1}$ to $\partial M$ along $\alpha_{1}$. Let $S_{2}$ be the first surface in a Haken decomposition for $M_{1}$. We may assume that $\partial S_{2}$ consists of $m_{2}$ copies of a nonseparating, simple, closed curve $\gamma \subset \partial M_{1}$ which do not intersect the two disks $\partial H_{1} \cap \partial M_{1}$. Since $\partial S_{2} \subset M$, we can identify $\gamma$ with $\alpha_{2}$. Note that $S_{2}$ may intersect $H_{1}$. If we cannot isotop the interior of $S_{2}$ to be disjoint from $H_{1}$, then we may assume that the intersection consists of $k$ disjoint disks $D_{i}$ on $S_{2}$. Moreover, we can assume that the disks all have the same sign of intersection with the cocore of $H_{1}$. If two disks had different signs of intersection, then we could find two adjacent such disks, remove the disks, and identify the boundaries to reduce the intersection of $S_{2}$ with $H_{1}$. Note that $\partial S_{1}$ consists of $n_{1}$ copies of the attaching curve for $H_{1}$. 
Therefore, we can take $k$ copies of $S_{1}$ and $n_{1}$ copies of $S_{2}$, remove the $k n_{1}$ disks $k S_{2} \cap H_{1}$ from $n_{1} S_{2}$, and use the $k n_{1}$ boundary components of $k S_{1}$ to cap off these boundary components, possibly reversing the orientation of $S_{1}$ if necessary. This operation shows that class $n_{2} \alpha_{2} \in K$, where $n_{2}=n_{1} m_{2}$. Attach another handle $H_{2}$ to $M_{1}$ along $\alpha_{2}$ to form a new manifold $M_{2}$. Continuing in this fashion, we find $n$ integers $n_{i}$ and an identification of $\partial M$ with $\Sigma$ such that $n_{i} \alpha_{i} \in K . \alpha_{i}$ are clearly linearly independent and thus generate $K$ since $\operatorname{dim}_{\mathbb{Q}}(K)=n[\mathbf{1 1}]$.

Given any 3-manifold with connected boundary, we identify $\partial M$ with the genus $n$ surface $\Sigma$ as specified in Lemma 7.2. We now describe the collection of curves $\Gamma \subset \partial M$ which will be the dividing set of a universally tight contact structure on $M$. Let $\gamma_{1}$ be a simple, closed curve homologous to $\alpha_{1}-2 \beta_{1}$ and let $\gamma_{i}$ be a simple, closed curve homolgous to $\alpha_{i}-\beta_{i}$ for $2 \leq i \leq n$. Finally, let $\gamma_{n+1}$ be a simple, closed curve homologous to $-\left(\gamma_{1}+\cdots+\gamma_{n}\right)$. Note that this collection of curves is diffeomorphic to the collection of curves shown in Figure 2.

Lemma 7.2. Let $M$ be any irreducible 3-manifold with connected boundary of nonzero genus. Then there exists a universally tight contact structure on $M$ such that $\partial M$ is convex and $\Gamma$ divides $\partial M$.

Proof. Let $(M, \gamma)$ be the sutured 3-manifold with annular sutures $s(\gamma)=\Gamma$. We will show that $(M, \gamma)$ is a taut sutured 3-manifold. We then invoke the result in [16] which says that $M$ also supports a universally tight contact structure with $\partial M$ convex and $\Gamma_{\partial M}=\Gamma$.

To prove that $(M, \gamma)$ is taut, it suffices to show that $M$ is irreducible, $R(\gamma)$ is Thurston norm-minimizing in $H_{2}(M, \gamma)$ among all other orientable surfaces in the same relative homology class, and $R(\gamma)$ is incompressible in $M$. By assumption, $M$ is irreducible. We now show $R(\gamma)$ is incompressible. Suppose not. Then the loop theorem $[\mathbf{1 1}]$ says that there exists an embedded disk $(D, \partial D) \subset(M, \partial M)$ such that $\partial D$ is homotopically nontrivial in $R(\gamma)$. Since $R(\gamma)$ consists of two planar surfaces and $\partial D$ is embedded, $\partial D$ must

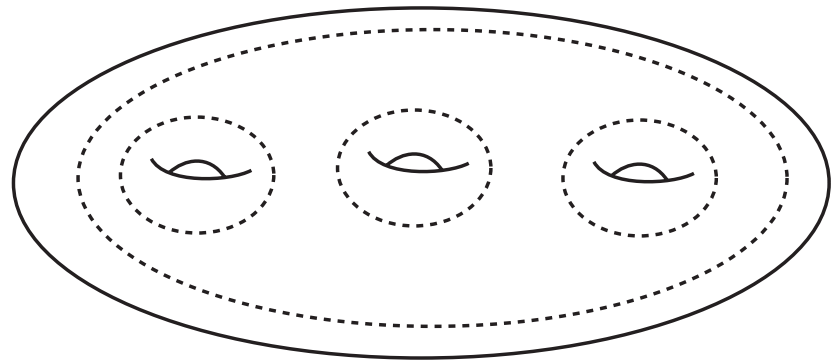

Figure 2. The collection of curves $\Gamma$ is diffeomorphic to the collection of curves shown above. 
also be homologous to $\pm\left(\gamma_{i_{1}}+\cdots+\gamma_{i_{j}}\right)$ where $1 \leq i_{1}, i_{j} \leq n$ are distinct. There exist $q_{i} \in \mathbb{Q}$ such that $\pm\left(\gamma_{i_{1}}+\cdots+\gamma_{i_{j}}\right)=q_{1} \alpha_{1}+\cdots+q_{n} \alpha_{n}$ since $\partial D$ is null homologous in $M$. Take the intersection pairing of each side with $\alpha_{i}$ to arrive at a contradiction.

We show that $R(\gamma)$ is Thurston norm-minimizing in $H_{2}(M, \gamma)$. Let $S=\cup S_{i}$ be any orientable surface homologous to $R(\gamma)$ in $H_{2}(M, \gamma)$. Without loss of generality, we assume that $\partial S \subset \operatorname{int}(A(\gamma))$. Fix an annulus $A(s) \subset A(\gamma)$ about the suture $s$ ( $s$ is a homologically nontrivial simple, closed curve in $A(s))$. Note that $\partial R(\gamma)$ intersects $A(s)$ in two oriented circles isotopic to $s$, where one comes from $R_{+}(\gamma)$ and the other comes from $R_{-}(\gamma)$. These circles must have the same orientation since the orientation of $R_{+}(\gamma)$ agrees with the orientation on $\partial M$ and the orientation on $R_{-}(\gamma)$ does not. Consider the intersection of $S$ with $A(s)$. If any two curves of $\partial S \cap A(s)$ have opposite orientation induced from $S$, then we can find two such curves which are adjacent. We then identify these curves and isotop them off of $\partial M$ to reduce the number of boundary components of $S$. We continue this procedure until $\partial S \cap A(s)$ consists of two curves with the same orientation, which agrees with the orientation of $\partial A(s)$ induced from $R(\gamma)$. Note that the orientation on and number of these remaining curves in $\partial S \cap A(s)$ are completely determined by the assumption that $[S]=[R(\gamma)]$ in $H_{2}(M, \gamma)$. To summarize, we may assume that $\partial S$ intersects each annulus of $A(\gamma)$ in exactly two essential curves with the same orientation induced from $S$, which agrees with the orientation of the boundary of the annulus induced $R(\gamma)$ (see Figure 3).

We assume that our curves are exactly as in Figure 2. Recall that $\partial R(\gamma)=$ $\cup_{j=1}^{n+1} \gamma_{j} \cup \cup_{j=1}^{n+1} \gamma_{j}$. Let $S_{i}$ be a component of $S$. We now show that $\partial S_{i}=$ $\cup_{j=1}^{n+1} \gamma_{j}$ or $\partial S_{i}=\cup_{j=1}^{n+1} \gamma_{j} \cup \cup_{j=1}^{n+1} \gamma_{j}$ as oriented manifolds. Note that $\partial S_{i}$ is the union of some subset of the oriented curves $\left\{\gamma_{1}, \gamma_{1}, \ldots, \gamma_{n+1}, \gamma_{n+1}\right\}$. Since $\partial S_{i} \subset K, \partial S_{i}=q_{1} \alpha_{1}+\cdots+q_{n} \alpha_{n}$. For $1 \leq j \leq n$, take the intersection

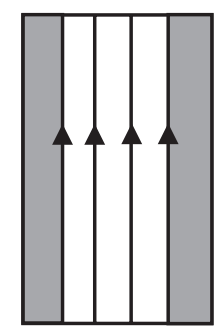

Figure 3. The white region is an annular suture. The gray region is $R(\gamma)$. The two vertical lines in the annulus are boundary curves of $S$ with orientation induced from $S$. The arrows on $\partial R(\gamma)$ denote the orientation induced from $R(\gamma)$. 
pairing of both sides of this expression with $\alpha_{j}$ to see that $\gamma_{j}$ and $\gamma_{n+1}$ must occur together (if they occur at all) in $\partial S_{i}$. This shows that $\partial S_{i}=\cup_{j=1}^{n+1} \gamma_{j}$ or $\partial S_{i}=\cup_{j=1}^{n+1} \gamma_{j} \cup \cup_{j=1}^{n+1} \gamma_{j}$. We say such surfaces are of type I or II, respectively. If $S_{i}$ is of type $I$, then $S$ consists of two such surfaces, and if $S_{i}$ is of type II, then $S=S_{i}$. In either case, $x(S) \geq x(R(\gamma))$, with equality when $S$ is planar.

7.1. Construction of the contact structures when $\partial M$ is connected. Let $(M, \eta)$ be the universally tight contact manifold given by Lemma 7.2. When we refer to well-behaved surfaces, we will mean well-behaved with respect to $\left(M, \eta ; \partial M, \lambda_{1},\left\{\alpha_{1}, \beta_{1}\right\}\right)$. Let $S_{1}$ be the first cutting surface in a hierarchy for $M$ with boundary $\alpha_{1}$. Recall that in the proof of Lemma 7.1, we chose $S_{1}$ so that $\partial S_{1}$ consists of $n_{1}$ copies of $\alpha_{1}$, so $S_{1}$ is well-groomed, as defined in [7]. Via the correspondence between sutured manifold decompositions and convex decompositions, we may assume that $S_{1}$ is the first cutting surface in a convex decomposition for $M$ and has $\partial$-parallel dividing curves (see [16]). Since $t b\left(S_{1}\right) \leq-2$, there is a bypass abutting $\partial M$ along $\alpha_{1}$. After attaching this bypass to $\partial M$, we have a $\Sigma \times[0,1]$ slice with convex boundary, where $\Sigma$ is a genus $n$ surface, $n$ is the genus of the boundary of $M$, and $\Sigma \times\{1\}=\partial M$. Let $(Y, \eta)$ denote this contact manifold. Note that after attaching this bypass, the dividing curves consist of $n(-1,1)$ curves on each of the tori summands and another simple, closed curve which is homologous to the sum of the other $n$.

We now construct an embedding of $Y$ into $S^{3}$ with the standard tight contact structure. Fix $g$ disjoint Darboux balls in $S^{3}$ labeled $B_{i}$, where $g$ is the genus of the slice $Y$. In $B_{1}$, we have a convex torus $T_{1}$ with slope -2 . One can find such a torus in $S^{3}$ and then remove a point from $S^{3}$ to get such a torus in $\mathbb{R}^{3}$. In each of the remaining $B_{i}$, we have a convex torus with slope -1 . On $T_{1}$, LeRP a curve $m_{1}$ which bounds a disk in $T_{1}$ containing a single arc of the dividing set. On each of the other $T_{i}$, LeRP a curve $l_{i}$ containing a disk in $T_{i}$ with a single arc of $\Gamma_{T_{i}}$ and LeRP a curve $m_{i}$ which is disjoint from $l_{i}$ and bounds a disk with a single arc of the same dividing curve that $l_{i}$ intersects. Now, remove the disks bounded by the $l_{i}$ and $m_{i}$ on $T_{i}$ and join $l_{i}$ to $m_{i+1}$ by a convex annulus $A_{i}$. This yields a convex genus $n$ surface. Inside $B_{1}$, we have a compressing disk for $T_{1}$. By the imbalance principle, there is a bypass along this compressing disk. Attaching this bypass yields the desired embedding of $Y$. Note that we can arrange for the sign of this bypass to agree with the sign of the bypass we attached to $\partial M$.

Fix a real number $r \in(-2,-1)$. Let $q_{i}$ be an infinite sequence of rationals constructed in Section 4 such that $q_{1}=-1$ and $q_{i} \neq r$.

Proposition 7.3. There exists a sequence $\Sigma_{i} \subset Y=\Sigma \times[0,1]$ of wellbehaved surfaces such that slope $\left(\Sigma_{i}\right)=q_{i}$ and $\Sigma_{1}=\Sigma \times\{0\}$. 
Proof. We will prove our results for the embedding of $Y \subset S^{3}$. LeRP copies $l_{i}$ of $\lambda_{1}$ on $\Sigma \times\{i\}$ such that $t b\left(l_{i}\right)=-1$. Let $A \subset Y$ be a convex annulus between $l_{0}$ and $l_{1}$. $l_{i}$ separates $\Sigma \times\{i\}$ into a punctured torus $P_{i}$ and a punctured genus $n-1$ surface. Cap off the $P_{i}$ in $S^{3}$ with convex disks $D_{i}$ to obtain tori $T_{i}$ such that slope $\left(T_{1}\right)=-2$ and slope $\left(T_{0}\right)=-1$. There exists an incompressible torus $T$ in the toric annulus bounded by the $T_{i}$ such that $\operatorname{div}(T)=1$ and $\operatorname{slope}(T)=q_{2}[\mathbf{1 3}]$. Let $d_{2}$ be a Legendrian divide on $T . d_{2}$ can be Legendrian isotoped within the toric annulus bounded by the $T_{i}$ so that it does not intersect $D^{2} \times[0,1]$, which we used to cap off the thickened punctured torus bounded by $P_{0} \cup P_{1} \cup A$. This can be seen working in a model for $D^{2} \times[0,1]$, a standard neighborhood of a Legendrian arc. Hence, there exists a Legendrian isotopy taking $d_{2}$ to a curve in $Y$ that is homologous to $a_{2} \alpha_{1}+b_{2} \beta_{1}$, where $q_{2}=b_{2} / a_{2}$. LeRP a curve $d_{2}^{\prime}$ in the same homology class on $\Sigma_{1}$ such that $d_{2}^{\prime} \cap \Gamma_{\Sigma_{1}}$ is minimal. Let $A_{2} \subset Y$ be a convex annulus between $d_{2}$ and $d_{2}^{\prime}$. By our choice of $d_{2}, \Gamma_{A_{2}} \cap d_{2}=\emptyset$ and $\Gamma_{A_{2}} \cap d_{2}^{\prime} \neq \emptyset$, so there exists a bypass along $d_{2}^{\prime}$. Attaching this bypass to $\Sigma_{1}$ yields $\Sigma_{2}$. Now, repeat the previous argument for $q_{3}$ and the slice bounded by $\Sigma_{2}$ and $\Sigma \times\{1\}$ to obtain $\Sigma_{3}$. These surfaces are well-behaved by construction.

Let $\Sigma_{i}$ be as in Proposition 7.3. Let $\left(Y_{i}, \eta\right)$ be the genus $n$ slice bounded by $\Sigma_{i}$ and $\Sigma_{i+1}$ in $Y$. Construct a contact structure $\eta$ on $\Sigma \times[0, \infty)$ by taking $\Sigma \times[i, i+1]$ to be $Y_{i}$. Let $\left(V, \eta_{r}\right)$ be obtained from $(M, \eta)$ by peeling off $Y \backslash \Sigma_{1}$ from $(M, \eta)$ and attaching $(\Sigma \times[0, \infty), \eta)$ in the obvious way. Note that $\left(V, \eta_{r}\right)$ is tight by construction since it embeds into $(M, \eta)$.

Lemma 7.4. Let $s, t \in(-2,-1)$. Then $\left(V, \eta_{s}\right)$ and $\left(V, \eta_{t}\right)$ are in the same isotopy class of contact structures.

Proof. There exists a convex surface $S \subset V$ such that $V \backslash S=V^{\prime} \cup S \times(0, \infty)$, where $V^{\prime}$ is diffeomorphic to $V$ and $\left.\eta_{s}\right|_{V^{\prime} \cup S}=\left.\eta_{t}\right|_{V^{\prime} \cup S}$. This follows from the construction of $\left(V, \eta_{s}\right)$ and $\left(V, \eta_{t}\right)$. We claim that $\left.\eta_{s}\right|_{S \times[0, \infty)}$ and $\left.\eta_{t}\right|_{S \times[0, \infty)}$ are isotopic relative to $S \times 0$. We can assume that $S \times[0,1)$ is a one-sided vertically invariant neighborhood of our convex surface $S \times 0$. Hence, in particular, $\eta_{t}$ and $\eta_{s}$ agree on $S \times[0,1)$. Form a new contact structure $\eta_{t}^{\lambda}$ as follows: Extend the vertically invariant neighborhood $S \times[0,1)$ of $\eta_{t}$ to $S \times[0, \lambda)$, and on $S \times[\lambda, \infty)$, take $\eta_{t}^{\lambda}$ to be $\left.\eta_{t}\right|_{S \times[1, \infty)}$. Define $\eta_{s}^{\lambda}$ similarly. By construction, $\eta_{t}^{\infty}=\eta_{s}^{\infty}$. Hence, $\left(V, \eta_{s}\right)$ and $\left(V, \eta_{t}\right)$ are in the same isotopy class of contact structures.

7.2. Proof of Theorem 1.2 and Theorem 1.3 when $\partial M$ is connected. In order to show that $V$ supports uncountably many tight contact structures that are not contactomorphic, we will first show that the $\left(V, \eta_{s}\right)$ are distinct up to proper isotopy. Theorem 1.2 then follows immediately since the mapping class group of any 3 -manifold with boundary is countable $([\mathbf{1 7}])$. To achieve this, we use the idea of the slope at infinity introduced in Section 3. 
Proposition 7.5. The net slope: $\mathcal{C}\left(\operatorname{Ends}\left(V, \eta_{s} ; \partial M\right)\right) \rightarrow \mathbb{R} \cup\{\infty\}$ is convergent, so the slope at infinity is defined. Moreover, the slope at infinity of $\eta_{s}$ is $s$ for all $s \in(-2,-1)$.

Proof. We first show that there is an $E \in \operatorname{Ends}\left(V, \eta_{s}\right)$ such that for all $F \subset E$, slope $(F) \leq s$. Choose $E \subset \operatorname{int}(Y)$. We will be now working in $S^{3}$. Let $F \subset E$ and suppose for contradiction that slope $(F)>s$. Then, there exists $\Sigma \in \mathcal{C}(E)$ such that $\operatorname{slope}(\Sigma)>s$. Let $\Sigma_{i}$ be the family of surfaces given by Proposition 7.3. There exists an $i$ such that $\Sigma$ is contained in the genus $n$ slice bounded by $\Sigma_{1}$ and $\Sigma_{i}$. LeRP a copy of $\lambda_{1}$ on $\Sigma, \Sigma_{1}$, and $\Sigma_{i}$ and cap off the punctured tori bounded by these curves with convex disks. This yields a toric annulus $T^{2} \times[0,1] \subset S^{3}$ which contains a convex, incompressible torus $T$ such that slope $(T)>\operatorname{slope}\left(T^{2} \times\{1\}\right)$. No such $T^{2} \times I$ can exist in $S^{3}$ (see [13]). Therefore, such a $\Sigma$ could not exist. Similarly, one can show that $\operatorname{slope}(F)<s$ leads to a contradiction. The existence of the family $\Sigma_{i}$ now implies that the slope at infinity is $s$.

By the proper isotopy invariance of the slope at infinity, there are uncountably many tight contact structures that are not properly isotopic, or even contact diffeomorphic, on $V$. This concludes the proof of Theorem 1.2 in the case of connected boundary. The proof of Theorem 1.3 is now immediate. For each $\eta_{s}$, simply choose a transverse curve in $V$ and introduce a Lutz twist. Since the contact structures is identical outside of a compact set, the slope at infinity is unchanged.

7.3. Proof of Theorem 1.2 and Theorem 1.3 when $\partial M$ is disconnected. Before proceeding with the proof, we will need the following technical result.

Lemma 7.6. For every nonzero, positive integer $n$, there exists an irreducible 3-manifold $M_{n}$ with connected, incompressible boundary of genus $n$.

Proof. Let $\Sigma_{g}$ be an orientable surface of genus $g$. If $n=2 m$, let $F \subset \Sigma_{n}$ be a once-punctured genus $m$ surface. Form a manifold $M_{n}$ by identifying $F \times\{0\}$ with $F \times\{1\}$ on $\Sigma_{n} \times[0,1]$. It is straightforward to show that $\Sigma_{n} \times\{0\}$ and $\Sigma_{n} \times\{1\}$ are incompressible in $M_{n}$. Using the incompressibility of these surfaces and the irreducibility of $\Sigma_{n} \times[0,1]$, it is routine to show that $M_{n}$ has incompressible boundary and is irreducible. If $n=2 m-1$, let $F \subset \Sigma_{m}$ be an annular neighborhood of a nonseparating, simple, closed curve. Form a manifold $M_{n}$ by identifying $F \times\{0\}$ with $F \times\{1\}$ on $\Sigma_{m} \times$ $[0,1]$. It is again straightforward to show that $\Sigma_{m} \times\{0\}$ and $\Sigma_{n} \times\{1\}$ are incompressible in $M_{n}$. Irreducibility and incompressibility of the boundary follow as before.

Let $\partial M=\cup_{i=1}^{n} S_{i}$ where $S_{i}$ are the connected components of $\partial M$ and $S_{1}$ is of nonzero genus. Let $S_{j}$ be any component different from $S_{1}$. If $S_{j}$ 
is compressible, compress it, and continue doing so until we have a collection of spheres and incompressible surfaces. We are now in the situation where every boundary component, besides possibly $S_{1}$, is incompressible or a sphere. Fill in each sphere with a ball and onto each incompressible component of genus $n$, excluding $S_{1}$ if it happens to be incompressible, glue in an irreducible manifold with connected, incompressible boundary of genus $n$ (such manifolds exist by Lemma 7.6). It is straightforward to show that the resulting manifold is irreducible since we are gluing irreducible manifolds (after filling any spheres in the boundary) along incompressible surfaces. Call the resulting manifold $M^{\prime}$. We are now in the case of connected boundary. Put a tight contact structure on $M^{\prime}$ as before and attach a bypass along $\alpha_{1}$ so that we have factored off a $\partial M^{\prime} \times[0,1]$ slice $Y$. Topologically, the closure of $M^{\prime} \backslash Y$ is again $M^{\prime}$. Without intersecting $Y$, remove each of the manifolds we glued in after perturbing the gluing surfaces to be convex. Reconstruct $M$ by gluing the boundary components back together along the compressing disks. To ensure that the resulting manifold is tight, choose the compressing disks to be convex with Legendrian boundary and with a single arc in the dividing set $[\mathbf{1 2}]$. We now have a tight contact structure on $M$ and a bypass layer $Y$ along $S_{1}$ which is identical to the case of connected boundary. To form the $\left(V, \eta_{s}\right)$, remove all the boundary components except for $S_{1}$ and construct the ends in $Y$ as before. The calculation of the slope at infinity is identical to the case of connected boundary. As in the case of con-

nected boundary, the proof of Theorem 1.3 is immediate after introducing a Lutz twist along a transverse curve in $V$.

\section{References}

[1] V. Colin, Un infinité de structures de contact tendues sur les variétés toroidales, Comment. Math. Helv. (2) (2001), 353-372.

[2] V. Colin, E. Giroux and K. Honda, On the coarse classification of tight contact structures, Proc. Symp. Pure Math. 71 Amer. Math. Soc. Providence, RI, 2003.

[3] Y. Eliashberg, Classification of contact structures on $\mathbb{R}^{3}$, Int. Math. Res. Not. (3) (1993), 87-91.

[4] Y. Eliashberg, Classification of overtwisted contact structures on 3-manifolds, Invent. Math. 98 (1989), 623-637.

[5] Y. Eliashberg, New invariants of open symplectic and contact manifolds, J. Amer. Math. Soc. 4 (1991), 513-520.

[6] J. Etnyre, Introductory lectures on contact geometry, Proc. Symp. Pure Math. 71 (2003), 81-107.

[7] D. Gabai, Foliations and the topology of 3-manifolds, J. Diff. Geom. (1983), 445-503.

[8] E. Giroux, Structures de contact en dimension trois et bifurcations des feuilletages de surfaces, Invent. Math. 141 (3) (2000), 615-689.

[9] E. Giroux, Structures de contact sur les variétés fibrées en cercles audessus d'une surface, Comment. Math. Helv. 76 (2) (2001), 218-262. 
[10] E. Giroux, Une infinité de structures de contact tendues sur une infinité de variétés, Invent. Math. 135 (3) (1999), 789-802.

[11] J. Hempel, 3-Manifolds, Princeton University Press, Princeton, N. J. University of Tokyo Press, Tokyo, 1976.

[12] K. Honda, Gluing tight contact structures, Duke Math. J. 115(3) (2002), 435-478.

[13] K. Honda, On the classification of tight contact structures I, Geom. Topol. 4 (2000), 309-368.

[14] K. Honda, On the classification of tight contact structures II, J. Diff. Geom. 55(1) (2000), 83-143.

[15] K. Honda, W. Kazez and G. Matić, Convex decomposition theory, Int. Math. Res. Not. (2) (2002), 55-88.

[16] K. Honda, W. Kazez and G. Matić, Tight contact structures and taut foliations, Geom. Topol. 4 (2000), 219-242.

[17] D. McCullough, 3-manifolds and their mappings, Seoul National University, Research Institute of Mathematics, Global Analysis Research Center, Lecture Notes Series, 26 Seoul, 1995, ii+83 pp.

[18] J. C. Sikorav, Quelques propriétés des plongements lagrangiens, Analyse globale et physique mathématique (Lyon, 1989), Mém. Soc. Math. France (N.S.), (46) (1991), 151-167.

[19] W. Thurston, A norm for the homology of 3-manifolds, Mem. Amer. Math. Soc. 59, (339) (1986), 99-130.

Department of Mathematics

University of Pennsylvania

209 South 33Rd StrEet

PhiladelPHiA, PA 19104-6395

E-mail address: james.j.tripp@gmail.com

Received 03/02/2006, accepted 05/03/2006

The author would like to thank John Etnyre, his advisor, for many helpful conversations and encouragement related to this work. He would also like to thank Stephan Schönenberger for reading drafts of this work. Finally, he would also like to thank Ko Honda, Will Kazez, and Gordana Matić for their comments and questions during his talk at the Georgia Topology Conference and the conversations that followed. 